\title{
Estrutura da comunidade de algas perifíticas em Eleocharis acutangula (Roxb.) Schult (Cyperaceae) em reservatório tropical raso, São Paulo, SP, Brasil
}

\author{
Vanessa Moraes Camargo ${ }^{1}$ e Carla Ferragut ${ }^{1,2}$
}

Recebido: 30.01.2013; aceito: 6.09.2013

\begin{abstract}
Periphytic algal community structure on Eleocharis acutangula (Roxb.) Schult (Cyperaceae) in a tropical shallow reservoir, São Paulo, São Paulo State, Brazil). The aim of this study was to evaluate the influence of seasonal variation of environmental conditions on the structural attributes of the periphyton community on Eleocharis acutangula (Roxb.) Schult (Cyperaceae) in the littoral zone of Ninféias Lake, São Paulo, São Paulo State, Brazil. The spring and summer were characterized by higher values of macrophyte cover, phytoplankton chlorophyll $a$, light, and phosphorus availability in the water. Periphyton biomass and algal density were negatively correlated with macrophyte cover and phytoplankton chlorophyll $a$. The species composition, algal classes, and descriptors changed in seasonal scale. We concluded that periphyton community structure on Eleocharis acutangula was strongly influenced by macrophyte cover and phytoplankton biomass. Availability of nutrientsin the water seems to play a minor role in community organization. The responses of the structural attributes of the periphyton showed a strong interrelationship with macrophytes and phytoplankton, which should be better understood in shallow tropical reservoirs.
\end{abstract}

Keywords: biomass, diversity, emergent macrophyte, species composition

RESUMO - (Estrutura da comunidade de algas perifíticas em Eleocharis acutangula (Roxb.) Schult (Cyperaceae) em reservatório tropical raso, São Paulo, SP, Brasil). O objetivo do presente estudo foi avaliar a influência da variação sazonal das condições ambientais sobre os atributos estruturais da comunidade de algas perifíticas em Eleocharis acutangula (Roxb.) Schult (Cyperaceae) na zona litorânea do Lago das Ninféias, São Paulo, SP, Brasil. A primavera e o verão caracterizaram-se pelos maiores valores de cobertura de macrófitas, clorofila-a do fitoplâncton, disponibilidade de luz e de fósforo na água. A biomassa e a densidade algal do perifíton foram correlacionadas negativamente com a cobertura de macrófitas e com a clorofila-a do fitoplâncton. A composição de espécies, classes algais e descritoras mudou em escala sazonal. Concluiu-se que a estrutura da comunidade de algas perifíticas em Eleocharis acutangula foi fortemente influenciada pela cobertura de macrófitas e biomassa do fitoplâncton. A disponibilidade de nutrientes na água parece ter menor importância sobre a organização da comunidade. As respostas dos atributos estruturais do perifíton evidenciaram a forte interrelação com macrófitas e fitoplâncton, a qual deve ser mais bem compreendida nos reservatórios tropicais rasos.

Palavras-chave: biomassa, composição de espécies, diversidade, macrófita emergente

\section{Introdução}

O perifiton é considerado um importante produtor primário em lagos, rios, riachos e planícies de inundação (Vadeboncoeur \& Steinman 2002). As algas são comumente os componentes mais abundantes do perifíton e possuem grande influência sobre estado nutricional da comunidade (Kahlert 1998, Frost et al. 2005). A estrutura da comunidade de algas perifíticas pode ser determinada por inúmeros fatores ambientais, os quais podem atuar em diferentes escalas (Stevenson 1997). Apesar do avanço do conhecimento sobre a ecologia do perifíton nas últimas décadas (Larned 2010), a identificação dos fatores ambientais que determinam a estrutura da comunidade de algas perifíticas é ainda um desafio para os pesquisadores, principalmente em região tropical.

Apesar da competição por recursos, as macrófitas aquáticas são substratos muito favoráveis para a colonização da comunidade perifítica, pois

1. Instituto de Botânica, Núcleo de Pesquisa em Ecologia, Caixa Postal 68041, 04045-972 São Paulo, SP, Brasil

2. Autor para correspondência: carlaferragut@yahoo.com.br 
podem fornecer ampla área de colonização e disponibilizar nutrientes durante os processos de excreção e senescência (Sand-Jensen \& Borum 1991, Burkholder 1996). As macrófitas enraizadas podem funcionar como uma bomba, retirando nutrientes do sedimento e disponibilizando para o perifíton (Wetzel 2001). A arquitetura e a forma biológica da macrófita também pode determinar a estruturação da comunidade perifítica, pois atuam diretamente sobre a disponibilidade de luz (sombreamento) e de área de colonização (Cattaneo et al. 1998, Santos et al. 2013). As macrófitas também podem influenciar negativamente a estrutura da comunidade perifítica por meio da liberação de substâncias alelopáticas (Erhard \& Gross 2006). O complexo perifíton-macrófita é descrito como uma unidade ecológica nos ecossistemas aquáticos rasos (Goldsborough et al. 2005) e, conjuntamente, podem indicar a qualidade da água (Kiss et al. 2003).

Conforme Chambers et al. (2008), o número de espécies de macrófitas aquáticas na região Neotropical é o mais elevado do planeta, mas o conhecimento sobre a estrutura da comunidade perifítica em macrófitas é ainda escasso. No Brasil, a variação sazonal da estrutura da comunidade de algas perifíticas foi descrita em macrófitas de diferentes formas de vida, como a flutuante Eichhornia azurea (Sw.) Kunth (Felisberto \& Rodrigues 2005), submersa enraizada Nymphaea spp. (Pellegrini \& Ferragut 2012), submersa livre Utricularia foliosa (Ferragut et al. 2011, Santos et al. 2013) e a emergente Panicum rivulare Trin. (Martins \& Fernandes 2007). Dentre as macrófitas emergentes, Eleocharis R.Br. é um dos gêneros de ampla distribuição nos ecossistemas aquáticos, pois pode ocorrer em brejos, cachoeiras, lagoas, lagos, margens de rios, pântanos, restingas e solos úmidos de locais abertos (Gil \& Bove 2007, Alves et al. 2010). Especificamente, Eleocharis acutangula (Roxb.) Schult é uma espécie nativa presente nos domínios fitogeográficos da Amazônia, Caatinga, Cerrado, Mata Atlântica, Pampa e Pantanal (Alves et al. 2010). Apesar da ampla distribuição da E. acutangula no Brasil, estudos sobre o perifiton nesta espécie são praticamente inexistentes. A estrutura da comunidade de algas perifíticas em Eleocharis vivipara e E. cellulosa foi descrita e associada à variação sazonal condições ambientais na planície de inundação da Flórida (Vymazal \& Richardson 1995, Havens et al. 1999).

Considerando a importância ecológica do complexo perifíton-macrófita para o funcionamento dos ecossistemas aquáticos rasos (Kiss et al. 2003) e a ampla distribuição do gênero Eleocharis no país (Alves et al. 2010), o objetivo do presente estudo foi avaliar a estrutura da comunidade de algas perifíticas na macrófita emergente Eleocharis acutangula (Roxb.) Schult. Especificamente, o estudo pretende avaliar a influência da variação sazonal das condições ambientais sobre os atributos estruturais do perifiton, visando identificar fontes de variabilidade da comunidade. De modo geral, o presente estudo contribuirá para o melhor entendimento da relação perifiton $\times$ macrófita na zona litorânea dos reservatórios tropicais rasos.

\section{Material e métodos}

Área de Estudo - O presente estudo foi realizado no Lago das Ninféias, um reservatório pequeno raso mesotrófico com profundidade máxima de 3,6 m, profundidade média de $1,32 \mathrm{~m}$ e tempo de residência médio de 7,2 dias (Bicudo et al. 2002). O Lago das Ninféias foi formado para fins paisagísticos pelo barramento do córrego Pirarungaua no Jardim Botânico de São Paulo, localizado no Parque Estadual das Fontes do Ipiranga (PEFI), dentro da malha urbana na região sudeste do Município de São Paulo (233'ㅇ'03,4"S, 46037'26,7"W).

Amostragem - As coletas para amostragem do perifíton em E. acutangula e das variáveis físicas, químicas e biológicas da água foram realizadas no outono (abril/2010), inverno (julho/2010), primavera (outubro/2010) e no verão (janeiro/2011). Os bancos de macrófitas aquática com presença de E. acutangula foram divididos em parcelas de $10 \mathrm{~m}^{2}$, das quais duas parcelas foram selecionadas aleatoriamente para a amostragem. Eleocharis acutangula foi encontrada em bancos pluriespecíficos de macrófitas aquáticas, os quais eram constituídos de Panicum repens L., Nymphaea spp., Utricularia foliosa L. e Eichhornia crassipes.

Variáveis Analisadas - Amostras de água da subsuperfície foram coletadas em cada parcela para determinação das variáveis físicas, químicas e biológicas. Foram analisadas as seguintes variáveis abióticas: transparência da água (profundidade do disco de Secchi), radiação subaquática (luxímetro Li-Cor LI-250A), temperatura, condutividade elétrica (condutivímetro Digimed), oxigênio dissolvido (Golterman et al. 1978), alcalinidade (Golterman \& Clymo 1971), pH (potenciômetro Digimed), formas de carbono inorgânico dissolvido, nitrito e nitrato (Mackeret et al. 1978), amônio (Solorzano 1969), ortofosfato e fósforo total dissolvido (PTD) 
(Strickland \& Parsons 1960), nitrogênio total (NT) e fósforo total (PT) (Valderrama 1981) e sílica solúvel reativa (Golterman et al. 1978). As amostras para a fração dissolvida dos nutrientes foram filtradas em filtro de fibra de vidro Whatman GF/F.

Em cada parcela amostrada, a cobertura de macrófita aquática, incluindo $E$. acutangula, foi determinada pelo método do quadrado (Thomaz et al. 2004). A biomassa fitoplanctônica foi determinada pela clorofila-a (corrigida da feofitina), utilizando etanol (90\%) como extrator (Marker et al. 1980, Sartory \& Grobblelar 1984).

O material perifítico foi removido do colmo de E. acutangula por meio de raspagem com escova de cerdas macias e jatos de água destilada. Todas as amostras foram replicadas $(n=2)$. Para determinação da clorofila-a, amostras foram filtradas em filtros de fibra de vidro (Whatman GF/F) na ausência direta de luz e para extração foi utilizado etanol conforme método descrito acima para o fitoplâncton.

Para a análise qualitativa das algas perifíticas, as amostras foram fixadas com solução de formalina a $4 \%$ e a identificação foi feita em microscópio binocular Axioscope Zeiss. O sistema de classificação adotado para classes e ordens foi o de van der Hoek et al. (1997) e para nível especifico utilizou-se literatura especializada. Amostras fixadas com lugol acético $0,5 \%$ foram utilizadas para contagem em microscópio invertido Zeiss Axiovert $(400 \times)$, de acordo com Utermöhl (1958). O tempo de sedimentação da câmara de contagem seguiu Lund et al. (1958). A contagem foi realizada em transectos e o limite de contagem foi estabelecido pela curva de rarefação de espécies e pela contagem mínima de 100 indivíduos da espécie mais abundante.

Para avaliação da estrutura, considerou-se como dominante a espécie com densidade superior a $50 \%$ da densidade total da amostra. Foram consideradas descritoras da comunidade perifítica apenas as espécies com contribuição acima de $5 \%$ da densidade total da amostra. As algas perifíticas foram classificadas de acordo com a forma de aderência ao substrato em prostradas, móveis, emaranhadas, heterotricosa (com projeções rizoidais) e pedunculada (Biggs et al. 1998).

A partir dos dados de densidade de espécies foram calculados os índices de diversidade e equitatividade de Shannon, e o índice de dominância de Simpson (Magurran 2004). A riqueza de espécies foi determinada pelo número total de táxons em cada amostra.

Tratamento estatístico - O coeficiente de correlação de Pearson $(r)$ foi usado para medir o grau de relação linear entre a biomassa e a densidade total da comunidade de algas perifíticas com a cobertura de macrófitas aquáticas e a clorofila-a do fitoplâncton $(\mathrm{r}>0.500, \mathrm{p}<0.05)$. A similaridade da composição de espécies da comunidade de algas perifíticas entre as estações do ano foi determinada pela Análise de Agrupamento, a qual foi calculada pela associação média (UPGMA) e índice de distância Bray-Curtis no programa estatístico PAST 2.13 (Hammer et al. 2001).

\section{Resultados}

Variáveis limnológicas - As condições limnológicas variaram sazonalmente no interior dos bancos pluriespecíficos de macrófitas. O resumo da variação das variáveis abióticas durante o período de estudo encontra-se na tabela 1. Considerando a disponibilidade de recursos para o perifiton, verificou-se que a maior disponibilidade de luz foi registrada na primavera e no verão. Contudo, a transparência da água chegou sempre até o fundo, devido à pequena profundidade dos pontos de amostragem $(0,3-0,6 \mathrm{~m})$. Quanto à disponibilidade de nutrientes dissolvidos na água, verificou-se que a maior concentração de amônio ocorreu no outono e a de nitrato no inverno. A concentração de ortofosfato esteve sempre abaixo do limite do método $\left(<4 \mu \mathrm{g} \mathrm{L}^{-1}\right)$, mas detectou-se aumento da concentração de PTD no verão e na primavera. Em média, os valores da razão molar N:P e NT:PT foram de 3,7 a 3,0 vezes maior no período seco (outono e inverno) do que no chuvoso (primavera e verão), respectivamente.

Fitoplâncton - Os maiores valores de clorofila-a do fitoplâncton no interior dos bancos pluriespecíficos de macrófitas ocorreram na primavera e no verão (tabela 1).

Macrófitas aquáticas - Nas parcelas amostradas, a maior cobertura total de macrófitas aquáticas foi registrada na primavera e no verão (98-92\%, respectivamente) e a menor no inverno (58\%) (figura 1).

Estrutura da comunidade de algas perifíticas - A biomassa e a densidade total de algas perifíticas apresentou forte variação sazonal e os maiores valores foram encontrados no inverno (figura 2a-b). A biomassa e a densidade total de algas perifíticas apresentaram significativa relação linear de dependência com a cobertura de macrófitas e com a clorofila-a do fitoplâncton ( $\mathrm{P}<0,000$; figura $3 \mathrm{a}-\mathrm{b})$. A correlação de Pearson mostrou que a relação entre os atributos do perifíton e a cobertura de macrófita e de clorofila-a do fitoplâncton foi negativa e significativa ( $\mathrm{P}<0,001$; figura 3). Assim, os atributos do perifiton 
apresentaram relação inversa com a cobertura de macrófitas e biomassa do fitoplâncton.

Foram identificados 134 táxons, os quais foram distribuídos em nove grupos taxonômicos: Bacillariophyceae, Chlorophyceae, Chrysophyceae, Cryptophyceae, Cyanophyceae, Dinophyceae, Euglenophyceae, Oedogoniophyceae, Zygnemaphyceae. Chlorophyceae e Zygnemaphyceae apresentaram o maior número de espécies (50 e 39, respectivamente).

Quanto à densidade relativa de classes algais, Bacillariophyceae, Chlorophyceae e Cyanophyceae apresentaram a maior participação na estrutura da comunidade perifítica (figura 4). A densidade relativa de Bacillariophyceae foi elevada em todas as estações do ano (41-73\%), exceto no verão quando Cyanophyceae foi dominante (56\%). Apesar da elevada densidade relativa de Bacillariophyceae no outono $(41 \%)$, verificou-se também o aumento da participação de Chrysophyceae e Chlorophyceae (15\% e $13 \%$, respectivamente).
Quanto à forma de aderência das algas ao substrato, a estrutura da comunidade algal apresentou elevada participação de formas sem estrutura de fixação durante todo o período de estudo (figura 5). O perifíton no outono apresentou maior participação das algas móveis (44\%) e das mucilaginosas $(22 \%)$. No inverno e na primavera houve maior participação das formas móveis $(44 \%$ e $56,5 \%$, respectivamente) e com motilidade moderada ( $22 \%$ e $11,5 \%$, respectivamente). Diferentemente, no verão as formas móveis permaneceram abundantes $(23,3 \%)$, mas as formas que crescem emaranhadas $(54,9 \%)$ ao substrato foram dominantes (figura 5).

Em relação densidade relativa das descritoras da comunidade perifítica (figura 6), constatou-se que Navicula cryptotenella (25\%) foi a espécie de maior contribuição para a estrutura no outono, seguida por Chromulina elegans (14\%) e Geitlerinema unigranulatum (10\%). No inverno Brachysira cf. vitrea representou $42 \%$ da densidade total, seguida por Brachysira brebissonii (12\%). Apesar da redução de 6,3 vezes da densidade de Brachysira cf. vitrea na

Tabela 1. Valores médios e desvio padrão $(\mathrm{n}=2)$ das variáveis abióticas e biológicas nos bancos de macrófitas com presença de Eleocharis acutangula (Roxb.) Schult (Cyperaceae) durante o período de estudo em reservatório tropical raso, São Paulo, SP, Brasil.

Table 1. Mean values and standard deviation $(\mathrm{n}=2)$ of abiotic and biological variables in macrophyte stands with presence of Eleocharis acutangula (Roxb.) Schult (Cyperaceae) during the study period in a tropical shallow reservoir, São Paulo, São Paulo State, Brazil).

\begin{tabular}{|c|c|c|c|c|}
\hline Variáveis & Outono & Inverno & Primavera & Verão \\
\hline Temperatura da água $\left({ }^{\circ} \mathrm{C}\right)$ & 22,2 & 18,5 & 21,7 & 24,8 \\
\hline Radiação subaquática $\left(\mu \mathrm{mol} \mathrm{S} \mathrm{S}^{-1} \mathrm{~cm}^{-2}\right)$ & $309,8( \pm 88,9)$ & $180,6( \pm 38,2)$ & $963,3( \pm 236,5)$ & $546,7( \pm 161,9)$ \\
\hline Transparência da água (m) & $0,42( \pm 0,06)$ & $0,30( \pm 0,00)$ & $0,38( \pm 0,08)$ & $0,62( \pm 0,1)$ \\
\hline Alcalinidade $\left(\mathrm{mEq} \mathrm{L}^{-1}\right)$ & $0,19( \pm 0,01)$ & $0,13( \pm 0,0)$ & $0,28( \pm 0,0)$ & $0,25( \pm 0,1)$ \\
\hline Condutividade elétrica $\left(\mu \mathrm{S} \mathrm{cm}^{-1}\right)$ & $58,4( \pm 2,2)$ & $56,9( \pm 1,2)$ & $57,3( \pm 1,4)$ & $50,5( \pm 0,6)$ \\
\hline Oxigênio dissolvido $\left(\mathrm{mg} \mathrm{L}^{-1}\right)$ & $4,4( \pm 0,3)$ & $6,3( \pm 0,9)$ & $4,0( \pm 0,1)$ & $3,4( \pm 0,1)$ \\
\hline Gás Carbônico livre (mg L-1) & $14,3( \pm 2,1)$ & $18,5( \pm 2,7)$ & $11,4( \pm 0,3)$ & $20,2( \pm 1,6)$ \\
\hline Potencial Hidrogeniônico & $6,2( \pm 0,1)$ & $6( \pm 0,0)$ & $6,4( \pm 0,0)$ & $6,1( \pm 0,0)$ \\
\hline Fósforo Total $\left(\mu \mathrm{g} \mathrm{L}^{-1}\right)$ & $34,8( \pm 8,3)$ & $10,8( \pm 5,6)$ & $13,8( \pm 2,3)$ & $18,3( \pm 5,2)$ \\
\hline Fósforo Total Dissolvido $\left(\mu \mathrm{g} \mathrm{L}^{-1}\right)$ & $<4$ & $<4$ & $6,0( \pm 0,8)$ & $6,0( \pm 0,8)$ \\
\hline $\mathrm{P}-\mathrm{PO}_{4}\left(\mu \mathrm{g} \mathrm{L}^{-1}\right)$ & $<4$ & $<4$ & $<4$ & $<4$ \\
\hline Nitrogênio Total $\left(\mu \mathrm{g} \mathrm{L}^{-1}\right)$ & $2549( \pm 120)$ & $1157( \pm 284)$ & $458( \pm 38)$ & $777( \pm 133)$ \\
\hline $\mathrm{N}-\mathrm{NO}_{2}\left(\mu \mathrm{g} \mathrm{L}^{-1}\right)$ & $12,3( \pm 1,3)$ & $6,9( \pm 2,9)$ & $8,8( \pm 0,9)$ & $17,5( \pm 0,4)$ \\
\hline $\mathrm{N}-\mathrm{NO}_{3}\left(\mu \mathrm{g} \mathrm{L}^{-1}\right)$ & $1258( \pm 305)$ & $1476( \pm 1047)$ & $268( \pm 101)$ & $824( \pm 256)$ \\
\hline $\mathrm{N}-\mathrm{NH}_{4}\left(\mu \mathrm{g} \mathrm{L}^{-1}\right)$ & $69( \pm 14)$ & $63( \pm 29)$ & $17,8( \pm 3,5)$ & $40,4( \pm 0,8)$ \\
\hline Silicato $\left(\mathrm{mg} \mathrm{L}^{-1}\right)$ & $2,2( \pm 0,1)$ & $3,4( \pm 226)$ & $2,0( \pm 21)$ & $3,2( \pm 57)$ \\
\hline Razão molar N:P inorgânica & $611,2( \pm 376)$ & $341,8( \pm 0,1)$ & $63,7( \pm 0,1)$ & $192,3( \pm 0,1)$ \\
\hline Razão molar NT:PT & $212,3( \pm 89)$ & $352,4( \pm 316)$ & $89,4( \pm 33)$ & $100,1( \pm 45)$ \\
\hline Clorofila- $a$ do Fitoplâncton & $7,2( \pm 0,7)$ & $6,0( \pm 4,9)$ & $9,7( \pm 1,2)$ & $9,2( \pm 4,3)$ \\
\hline
\end{tabular}




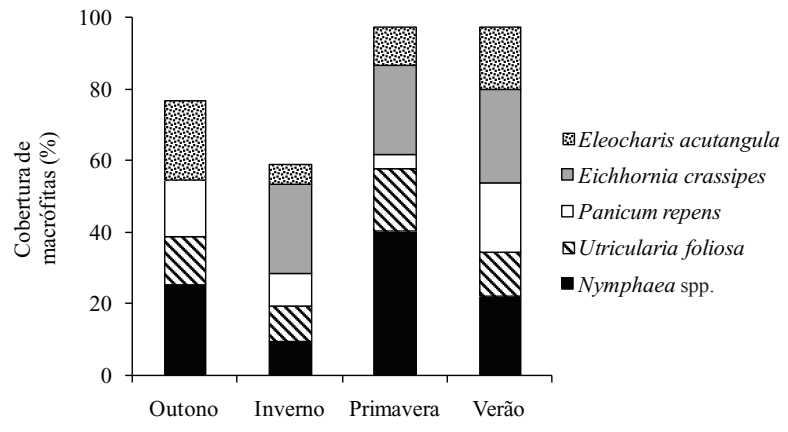

Figura 1. Variação sazonal da cobertura de macrófitas aquáticas nos pontos de amostragem no período de estudo em reservatório tropical raso, São Paulo, SP, Brasil.

Figure 1. Seasonal variation of aquatic macrophyte cover in the plots sampled during study period in a tropical shallow reservoir, São Paulo, São Paulo State, Brazil.

primavera, esta espécie foi dominante na estrutura da comunidade (56\%). Diferentemente, no verão a espécie de maior contribuição foi Geitlerinema unigranulatum (45\%), seguida por Navicula cryptotenella (8\%) e Pseudanabaena galeata (6\%).

A comunidade perifítica apresentou os maiores valores de riqueza e diversidade de espécies no outono e no inverno (figura 7a-b). A maior diversidade de espécies foi registrada no outono, mas a amplitude dos dados foi pouco acentuada entre as estações do ano $(3,1-3,9)$. A uniformidade de distribuição das espécies apresentou pouca ou nenhuma variação entre as estações do ano (figura 7c). $\mathrm{O}$ coeficiente de variação calculado para os dados de riqueza foi de $39 \%$ e para os de diversidade de $12 \%$. Diferentemente, a dominância de espécies foi maior na primavera e no verão (figura $7 \mathrm{~d}$ ).

a

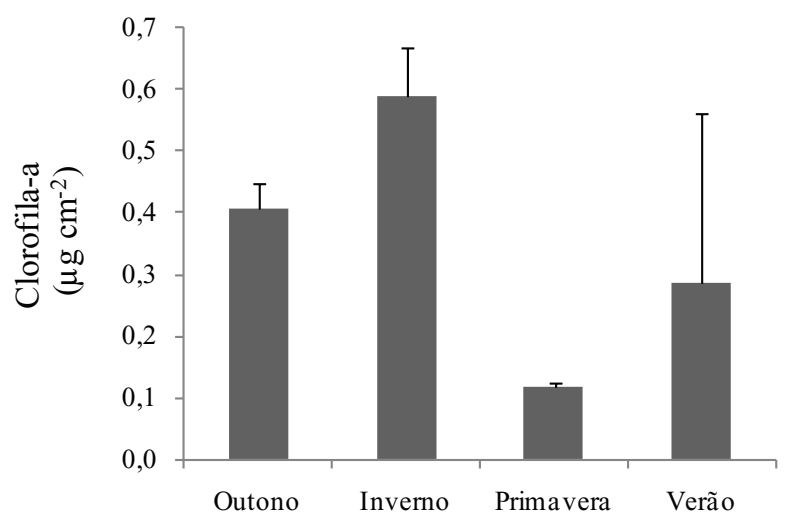

A análise de agrupamento mostrou a formação de dois grandes grupos ao nível de $15 \%$ de similaridade (figura 8): período seco (outono e inverno) e período chuvoso (primavera e verão). A similaridade na composição de espécies foi de $38 \%$ entre o outono e o inverno, e de $19 \%$ entre primavera e verão. O coeficiente cofenético de 0,9159 mostrou que o agrupamento foi consistente.

\section{Discussão}

O presente estudo mostrou que o perifíton em Eleocharis acuntangula desenvolveu-se dentro de uma ampla variação sazonal das condições ambientais na zona litorânea do Lago das Ninféias. As parcelas amostradas no outono e no inverno caracterizaram-se pela menor cobertura de macrófitas, menor biomassa (clorofila-a) do fitoplâncton, menor disponibilidade de luz (radiação e transparência) e alta disponibilidade de $\mathrm{N}$ na água. Situação oposta foi observada nas parcelas amostradas na primavera e no verão. Com base na razão N:P de Redfield da água, estudos anteriores no Lago das Ninféias descreveram o fósforo como nutriente limitante da comunidade algal (Fermino et al. 2011, Fonseca \& Bicudo 2011). Presentemente, a razão molar N:P e a NT:PT da água também indicaram condição P-limitante durante o período de estudo, mas o potencial de limitação algal foi substancialmente reduzido no período chuvoso (verão e primavera).

A maior biomassa e densidade total de algas perifíticas em $E$. acutangula ocorreram no outono e inverno, indicando que as condições ambientais foram favoráveis ao desenvolvimento do perifíton.

$\mathrm{b}$

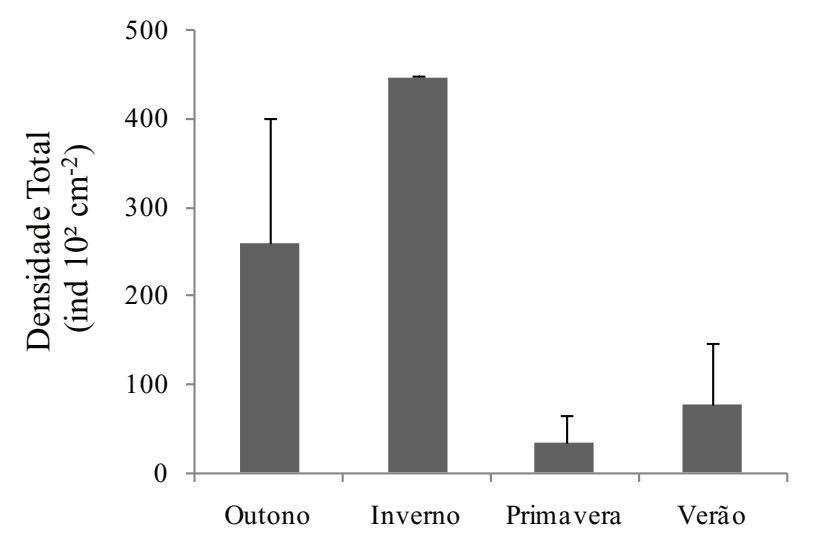

Figura 2. Biomassa (clorofila-a) (a) e densidade total (b) de algas perifíticas em Eleocharis acutangula (Roxb.) Schult (Cyperaceae) durante o período de estudo em reservatório tropical raso, São Paulo, SP, Brazil.

Figure 2. Periphyton algal biomass (chlorphyll a) (a) and total density (b) on Eleocharis acutangula (Roxb.) Schult (Cyperaceae) during the study period in a tropical shallow reservoir, São Paulo, São Paulo State, Brazil. 
Contudo, no outono e no inverno detectou-se a menor disponibilidade de fósforo dissolvido, maior desbalanceamento estequiométrico entre $\mathrm{N}$ e $\mathrm{P}$ e a menor transparência da água. A redução destes recursos deveria ter atuado negativamente sobre os atributos estruturais do perifiton, pois ambos os recursos podem limitar o crescimento algal. Estes resultados mostraram que outros fatores também podem atuar sobre a estrutura da comunidade. Além de fortes competidores por recursos (Vadeboncoeur \& Steinman 2002), as macrófitas e o fitoplâncton podem atuar negativamente sobre o perifíton por meio da redução da disponibilidade de luz por sombreamento (Kiss et al. 2003, Borduqui \& Ferragut 2012). Assim, acreditamos que o aumento da cobertura de macrófitas e da biomassa fitoplanctônica no período seco exerceu influência negativa sobre desenvolvimento do perifíton em $E$. acutangula.

a
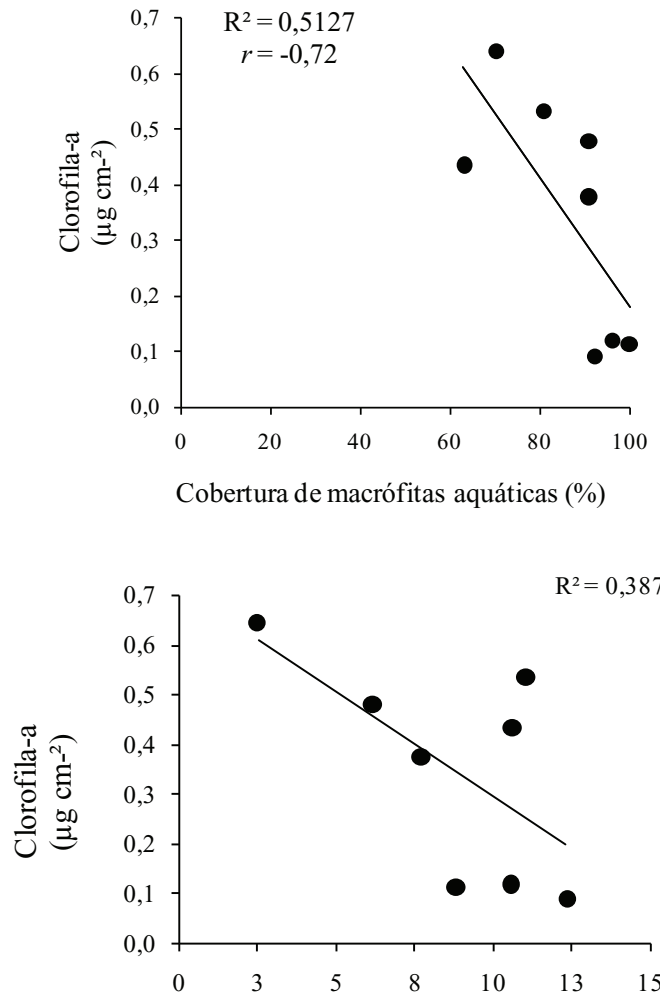

Clorofila-a do Fitoplâncton ( $\left.\mu \mathrm{g} \mathrm{L}^{-1}\right)$
Em relação aos substratos vivos, outro fator que pode reduzir ou inibir o crescimento do perifíton é a liberação de substâncias alelopáticas pela macrófita hospedeira (Burkholder 1996) cuja produção pode variar em escala sazonal (Whittaker \& Feeny 1971). Nakai et al. (1999) relataram a ausência de efeito alelopático sobre o crescimento de cianobactérias e clorofíceas em Eleocharis acicularis. No presente estudo nenhum indício de inibição do desenvolvimento do perifíton foi observado em E. acutangula, pois a biomassa algal máxima $\left(0,6 \mu \mathrm{g} \mathrm{cm}^{-2}\right)$ esteve dentro da amplitude $\left(0,1-2,3 \mu \mathrm{g} \mathrm{cm}^{-2}\right)$ registrada para o perifíton em outras espécies de macrófitas (p. ex. Felisberto \& Rodrigues 2005, Ferragut et al. 2011, Pellegrini \& Ferragut 2012). Além disso, a diversidade de espécies foi elevada em todas as estações do ano $(3,1-3,9) \mathrm{e}$, ainda, esteve dentro da amplitude $(2,8-4,6)$ reportada o perifíton nos reservatórios do PEFI (Vercellino \&

b

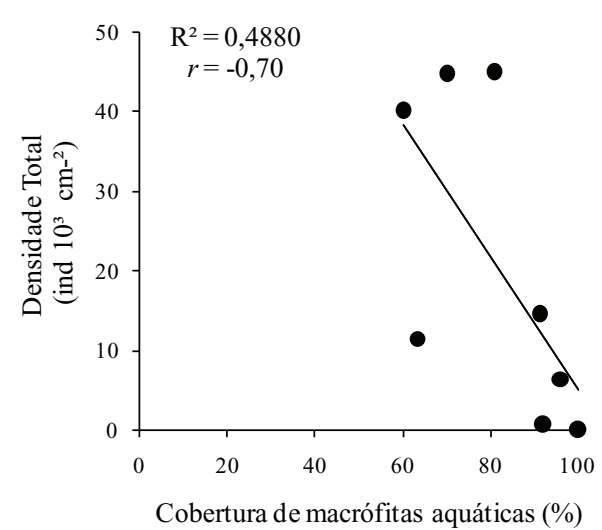

d

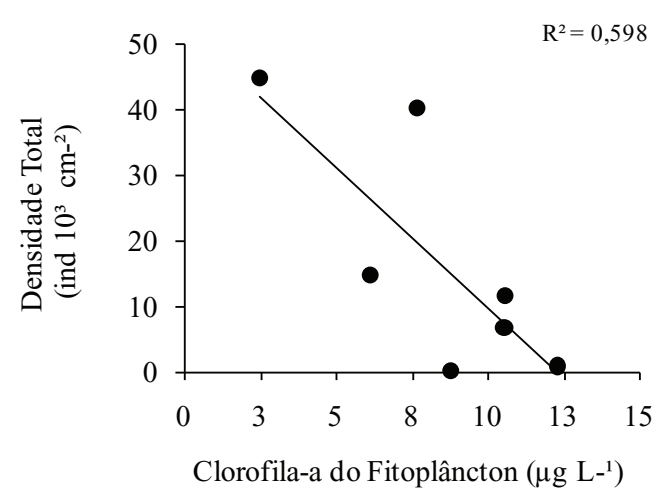

Figura 3. Diagrama de dispersão da biomassa e densidade total de algas perifíticas com a cobertura de macrófitas (a-b) e com a clorofila-a do fitoplâncton (c-d) em reservatório tropical raso, São Paulo, SP, Brasil. O símbolo $r$ indica o coeficiente da correlação de Pearson e o $\mathrm{R}^{2}$ indica o coeficiente de determinação da regressão linear simples.

Figure 3. Scatterplot of periphyton algal biomass and total density with macrophyte cover (a-b) and the phytoplankton chlorophyll $a$ (c-d) in a tropical shallow reservoir, São Paulo, São Paulo State, Brazil. The symbol $r$ indicates the Pearson correlation coefficient and $\mathrm{R}^{2}$ indicates the coefficient of determination of linear regression. 
Bicudo 2006, Borduqui \& Ferragut 2012, Pellegrini \& Ferragut 2012).

A similaridade da composição de espécies de algas perifíticas em $E$. acutangula mudou sazonalmente, evidenciando a sensibilidade da estrutura às alterações ambientais. Em região tropical, estudos mostraram a sazonalidade como determinante das mudanças estruturais da comunidade de algas perifíticas em substrato natural (p. ex. Vymazal \& Richardson 1995 , Fonseca \& Rodrigues 2005, Pellegrini \& Ferragut 2012, Santos et al. 2013). As mudanças estruturais também foram observadas em nível de classes algais e espécies descritoras. Bacillariophyceae apresentou elevada participação na estrutura da comunidade perifítica durante o período de estudo, exceto no verão quando houve dominância de Cyanophyceae. Em Eleocharis vivipara e Eleocharis cellulosa na planície de inundação da Flórida, Vymazal \& Richardson (1995) relataram a dominância de diatomáceas no inverno e na primavera e de cianofíceas no verão. Nos reservatórios do PEFI, a dominância de diatomáceas no perifíton é comumente reportada no período seco (p. ex. Borduqui et al. 2008, Pellegrini \& Ferragut 2012), o qual caracteriza-se pela ocorrência de mistura da coluna da água (Bicudo et al. 2002, Fonseca \& Bicudo 2011). Conforme Willén (1991) o aumento da turbulência da água favorece as diatomáceas, pois diminui a taxa de sedimentação da maioria das espécies. As diatomáceas de maior participação na estrutura da comunidade perifítica foram Navicula cryptotenella (outono) e Brachysira cf. vitrea (inverno e primavera). Navicula cryptotenella foi

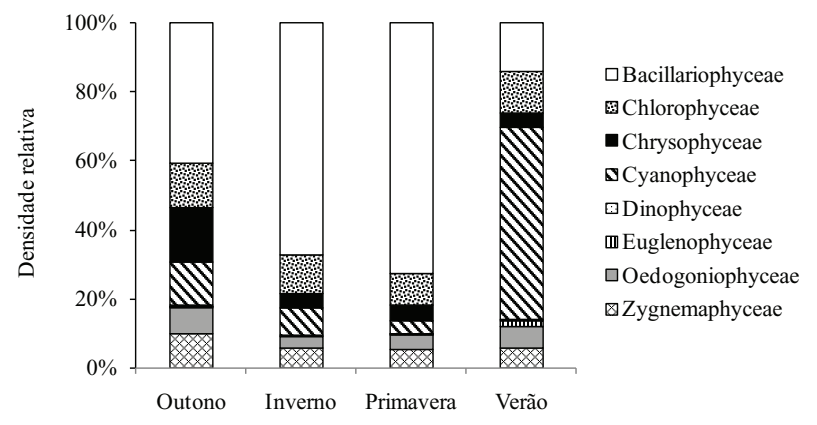

Figura 4. Densidade relativa de classes algais do perifiton em Eleocharis acutangula (Roxb.) Schult (Cyperaceae) durante o período de estudo em reservatório tropical raso, São Paulo, SP, Brasil.

Figure 4. Relative density of periphytic algal classes on Eleocharis acutangula (Roxb.) Schult (Cyperaceae) during the study period in a tropical shallow reservoir, São Paulo, São Paulo State, Brazil. reportada como descritora do perifíton em Nymphaea no período seco (Pellegrini \& Ferragut 2012) e, conforme Salomoni \& Torgan (2008), possuem ampla tolerância sapróbica. Diferentemente, no verão houve dominância da cianofícea filamentosa Geitlerinema unigranulatum. Esta espécie é bastante comum nos reservatórios tropicais e crescem em massas sobre o sedimento ou macrófitas aquáticas, principalmente em ambientes não poluídos (Komárek \& De Paiva Azevedo 2000).

A estrutura do perifíton apresentou elevada participação de algas móveis e com motilidade moderada em todas as estações do ano. De acordo com Fonseca \& Bicudo (2011), o fitoplâncton do Lago das Ninféias é representado por algas nanoplanctônicas flageladas, as quais possuem a motilidade como vantagem competitiva no heterogêneo ambiente criado pelas macrófitas aquáticas. Independentemente do habitat, a mobilidade das algas consiste em vantagem adaptativa de sobrevivência, pois permite o acesso a diferentes fontes de recursos (Happey-Wood 1988). As algas estão sempre estrategicamente posicionadas na matriz perifítica para utilizar, de modo eficiente, os recursos nutricionais disponíveis no substrato e na coluna d'água (Burkholder 1996).

Em conclusão, a estrutura da comunidade de algas perifíticas em Eleocharis acutangula foi influenciada pela escala sazonal de variação das condições ambientais, as quais foram mais favoráveis ao desenvolvimento da comunidade no período seco. A disponibilidade de nutrientes na água parece ter importância menor sobre a organização da

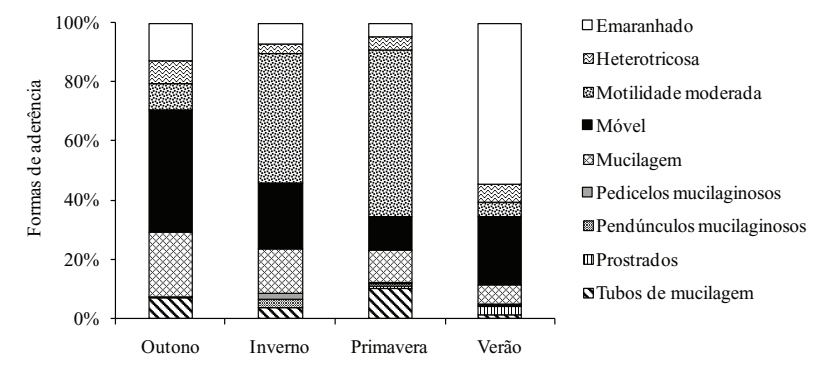

Figura 5. Densidade relativa das formas de aderência das algas perifiticas ao substrato, Eleocharis acutangula (Roxb.) Schult (Cyperaceae), durante o período de estudo em reservatório tropical raso, São Paulo, SP, Brasil.

Figure 5. Relative density of periphytic algal adherence forms on the substrate, Eleocharis acutangula (Roxb.) Schult (Cyperaceae), during the study period in a tropical shallow reservoir, São Paulo, São Paulo State, Brazil. 


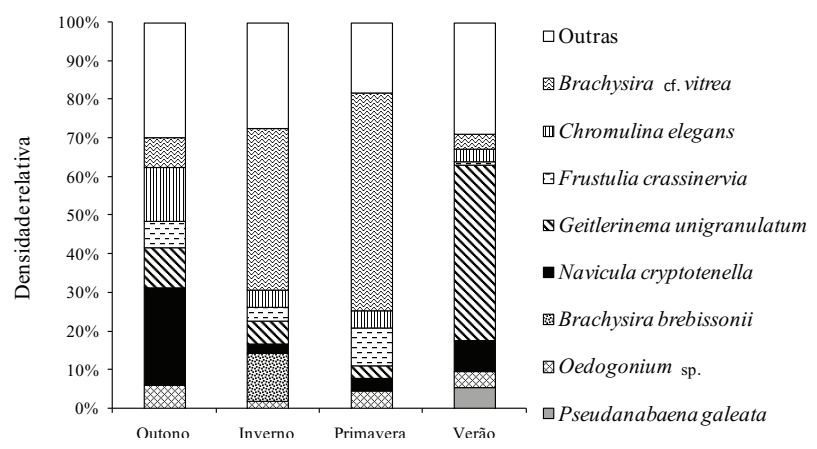

Figura 6. Densidade das espécies descritoras da comunidade perifitica em Eleocharis acutangula (Roxb.) Schult (Cyperaceae) durante o período de estudo em reservatório tropical raso, São Paulo, SP, Brasil.

Figure 6. Density of species descriptors on Eleocharis acutangula (Roxb.) Schult (Cyperaceae) during the study period in a tropical shallow reservoir, São Paulo, São Paulo State, Brazil.
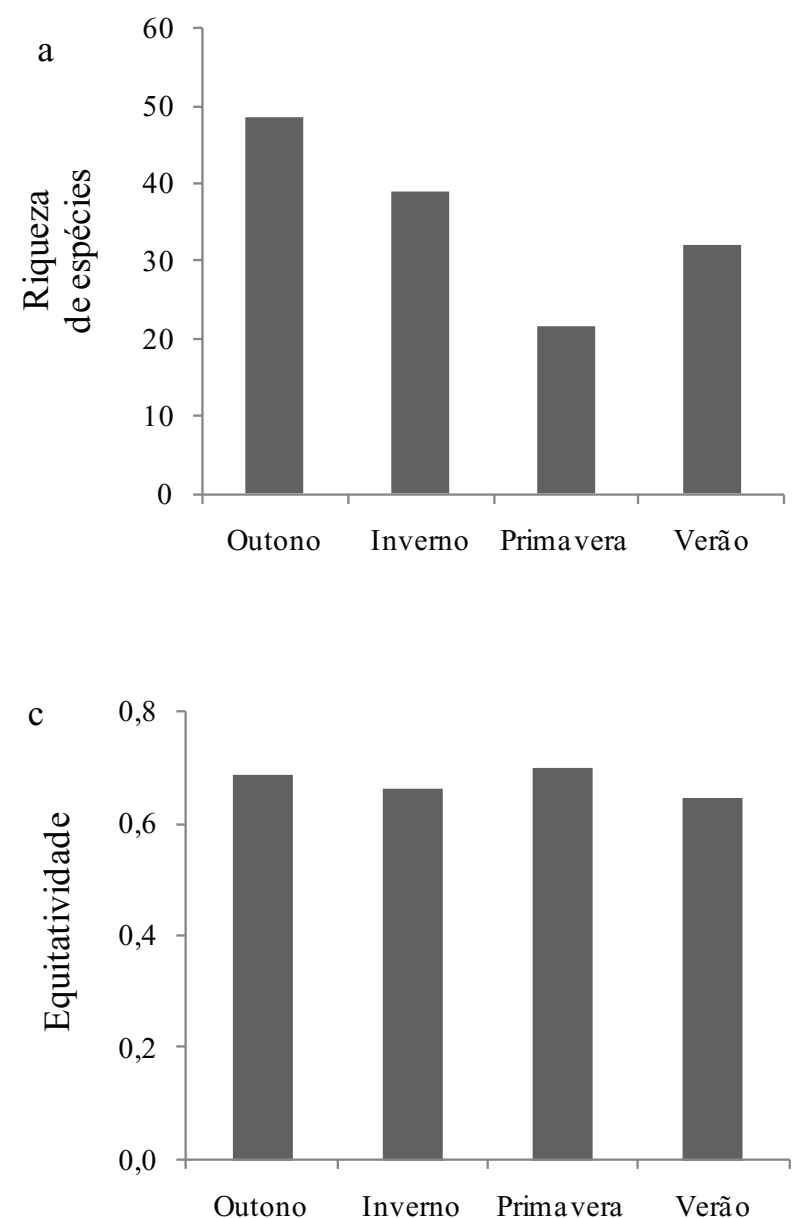

comunidade algal em E. acutangula. A cobertura de macrófitas e biomassa do fitoplâncton exerceram forte influência sobre a estrutura da comunidade de algas perifíticas. Além da interação competitiva, tais fatores devem, muito provavelmente, atuar negativamente sobre a disponibilidade de luz para o perifíton. Finalmente, as respostas dos atributos estruturais do perifiton evidenciaram a forte interrelação entre macrófitas, fitoplâncton e perifíton, a qual deve ser mais bem compreendida nos reservatórios tropicais rasos. Ressalta-se, ainda, que novas investigações são necessárias para o melhor entendimento do complexo perifíton $\times$ Eleocharis, principalmente, sobre a influência da macrófita no estado nutricional do perifíton, o qual pode atuar sobre a organização das assembleias algais.
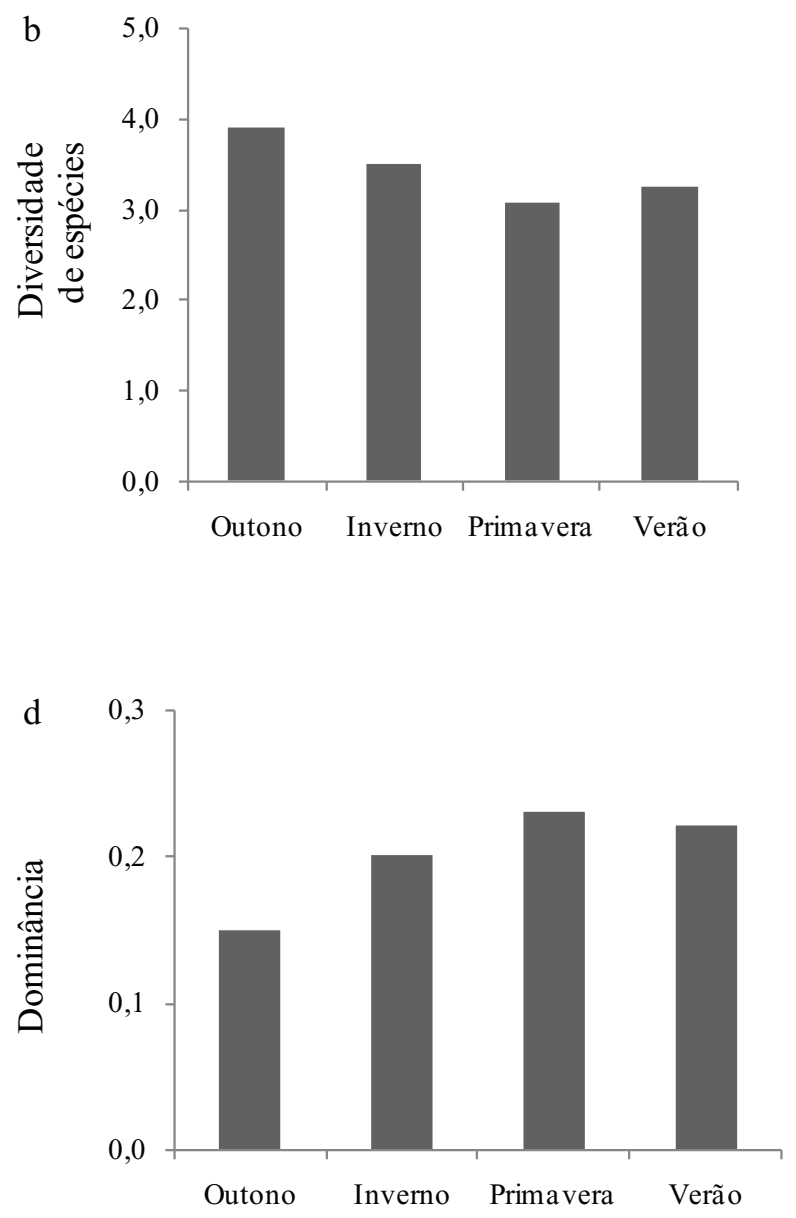

Figura 7. Riqueza (a), diversidade de espécies (b), equitatividade (c) e dominância (d) das algas perifíticas em Eleocharis acutangula (Roxb.) Schult (Cyperaceae) durante o período de estudo em reservatório tropical raso, São Paulo, SP, Brasil.

Figure 7. Richness (a), species diversity (b), evenness (c), and dominance (d) of periphytic algal on Eleocharis acutangula (Roxb.) Schult (Cyperaceae) during the study period in a tropical shallow reservoir, São Paulo, São Paulo State, Brazil. 


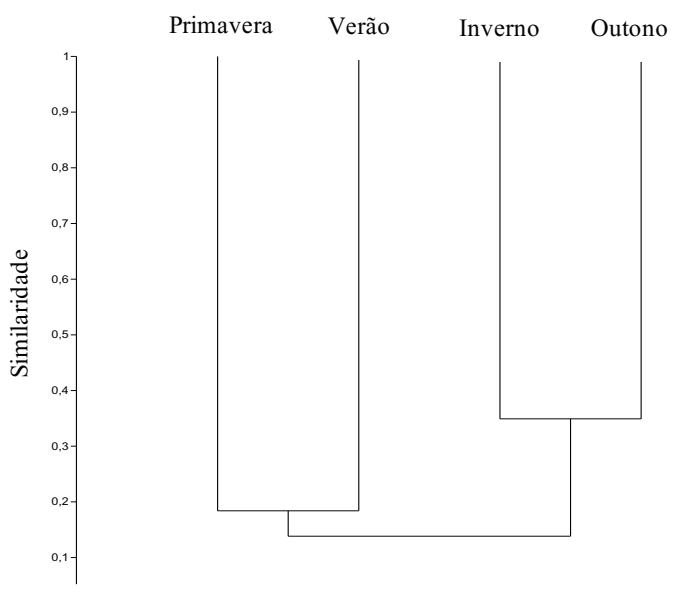

Figura 8. Similaridade da composição de espécies da comunidade de algas perifíticas em Eleocharis acutangula (Roxb.) Schult (Cyperaceae) entre as estações do ano (distância de Bray-Curtis) em reservatório tropical raso, São Paulo, SP, Brasil.

Figure 8 . Similarity of the periphytic algae composition on Eleocharis acutangula (Roxb.) Schult (Cyperaceae) between the seasons (Bray-Curtisdistance) in a tropical shallow reservoir, São Paulo, São Paulo State, Brazil.

\section{Agradecimentos}

Os autores agradecem à FAPESP, Fundação de Amparo à Pesquisa do Estado de São Paulo, pela bolsa de iniciação científica concedida à primeira autora (Processo no 2012/09027-6) e ao apoio financeiro para realização do projeto (Processo 2009/52253-4) coordenado pela segunda autora. Os autores agradecem à Dra. Cintia Kameyama do Núcleo de Pesquisas Curadoria do Herbário do Instituto de Botânica de São Paulo, SMA, pela a identificação da macrófita Eleocharis acutangula (Roxb.) Schult (Cyperaceae).

\section{Literatura citada}

Alves, M., Araújo, A.C., Hefler, S.M., Trevisan, R. \& Silveira, G.H. 2010. Cyperaceae. In: Lista de Espécies da Flora do Brasil. Jardim Botânico do Rio de Janeiro. http://floradobrasil.jbrj.gov.br (acesso em 07.04.2013).

Bicudo, C.E.M., Carmo, C.F., Bicudo, D.C., Henry, R., Pião, A.C.S., Santos, C.M. \& Lopes, M.R.M. 2002. Morfologia e morfometria de três reservatórios no PEFI. In: D.C Bicudo, M.C Forti \& C.E.M Bicudo (eds.) Parque Estadual das Fontes do Ipiranga (PEFI): unidade de conservação que resiste à urbanização de São Paulo. Secretaria do Meio Ambiente do Estado de São Paulo, São Paulo, pp. 143-160.

Biggs, B.J.F., Stevenson, R.J. \& Lowe, R.L. 1998. A habitat matrix conceptual modes for stream periphyton. Archiv für Hydrobiologie 143: 21-56.
Borduqui, M., Ferragut, C. \& Bicudo, C.E.M. 2008. Chemical composition and taxonomic structure vertical and seasonal variation of periphyton community in a shallow hypereutrophic reservoir (Garças Reservoir, São Paulo, Brazil). Acta Limnologica Brasiliensia 20: 381-392.

Borduqui, M. \& Ferragut, C. 2012. Factors determining periphytic algae succession in a tropical hypereutrophic reservoir. Hydrobiologia 683: 109-122.

Burkholder, J.M. 1996. Interaction of benthic algae with their substrata. In: R.J Stevenson, M. L. Bothwell \& R.L. Lowe (eds). Algal Ecology: freshwater benthic ecosystems. Academic Press, San Diego, pp. 253-298.

Cattaneo, A., Galanti, G., Gentinetta, S. \& Romo, S. 1998. Epiphytic algae and macroinvertebrates on submerged and floating-leaved macrophytes in an Italian lake. Freshwater Biology 39: 725-740.

Chambers, P.A., Lacoul P., Murphy K. J. \& Thomaz, S. M. 2008. Global diversity of aquatic macrophytes in freshwater. Hydrobiologia 595: 9-26.

Erhard, D. \& Gross, E.M. 2006. Allelopathic activity of Elodea canadensis and Elodea nuttallii against epiphytes and phytoplankton. Aquatic Botany 85: 203-211.

Felisberto, S.A. \& Rodrigues, L. 2005. Periphytic community of reservoirs cascade in the Paranapanema river, Brazil. Acta Scientiarum, Biological Sciences 27: 215-223.

Fermino, S.F., Bicudo, C.E.M. \& Bicudo, D.C. 2011. Seasonal influence of nitrogen and phosphorus enrichment on the floristic composition of the algal periphytic community in a shallow tropical, mesotrophic reservoir (São Paulo, Brazil). Oecologia Australis 15: 476-493.

Ferragut, C., Rodello, A.F. \& Bicudo, C.E.M. 2011. Seasonal variability of periphyton nutrient status and biomass on artificial and natural substrates in a tropical mesotrophic reservoir. Acta Limnologica Brasiliensia 22: 397-409.

Fonseca, B.M. \& Bicudo, C.E.M. 2011. Phytoplankton seasonal and vertical variations in a tropical shallow reservoir with abundant macrophytes (Ninféias Pond, Brazil). Hydrobiologia 665: 229-245.

Fonseca, I.A. \& Rodrigues, L. 2005. Comunidade de algas perifíticas em distintos ambientes da planície de inundação do alto rio Paraná. Acta Scientiarum, Biological Sciences 27: 21-28.

Frost, P.C., Hillebrand, H. \& Kahlert, M. 2005. Low algal carbon content and its effect on the C:P stoichiometry of periphyton. Freshwater Biology 50: 1800-1807.

Gil, A.S.B. \& Bove, C.P. 2007. Eleocharis R. Br. (Cyperaceae) no Estado do Rio de Janeiro, Brasil. Biota Neotropica 7: 1-31.

Goldsborough, L.G., McDougal, R.L. \& North, A.K. 2005. Periphyton structure, diversity and colonization. $I n$ : M.E. Azim, M.C.M. Beveridge, A.A. Van Dam, \&. M.C.J. Verdegem, (eds.), Periphyton: Ecology, exploitation and management. CABI Publishing, Cambridge, pp. 71-83. 
Golterman, H.L. \& Clymo, R.S. 1971. Methods for chemical analysis of freshwaters. Ed. Oxford and Edinburg. Blackwell Scientific Publications. International Biological Programmer, Oxford.

Golterman, H.L., Clymo, R.S. \& Ohmstad, M.A.M. 1978. Methods for physical and chemical analysis of freshwaters. 2 ed. Blackwell Scientific Publications. International Biological Program, Oxford.

Hammer, O., Harper, D.A.T. \& Ryan, P.D. 2001. PAST: Paleontological Statistics Software Package for Education and Data Analysis. Palaeontologia Electronica 4: 1-9.

Happey-Wood, V.M. 1988. Ecology of freshwater planktonic green algae. In: Growth and reproductive strategies of freshwater phytoplankton. C.D. Sandgreen (ed). Press Syndicate of the University of Cambridge, Cambridge, pp. 175-226.

Havens, K.E., East, T.L., Hwang, S.J., Rodwsky, A.J., Sharfstein, B. \& Steinman, A.D. 1999. Algal responses to experimental nutrient addition in the littoral community of a subtropical lake. Freshwater Biology 42: 329-344.

Kahlert, M. 1998. C:N:P ratios of freshwater benthic algae. Archiv für Hydrobiologie, Special Issue Advances in Limnology 51: 105-114.

Komárek, J. \& De Paiva Azevedo, M.T. 2000. Geitlerinema unigranulatum, a common tropical cyanoprokaryote from freshwater reservoirs in Brazil. Archiv für Hydrobiologie, Supplementband, Algological studies 134: 39-52.

Kiss, M.K., Lakatos, G., Borics, G., Gido, Z. \& Deak, C. 2003. Littoral macrophyte-periphyton complexes in two Hungarian shallow waters. Hydrobiologia 506-509: 541-548.

Larned, S.T. 2010. A prospectus for periphyton: recent and future ecological research. Journal of the North American Benthological Society 29:182-206.

Lund, J.W.G., Kipling, C. \& Le Cren, E.D. 1958. The inverted microscope method of estimating algal number and the statistical basis of estimating by counting. Hydrobiologia 11:143-170.

Magurran, A.E. 2004. Measuring Biological Diversity. Blackwell Science Ltd, Oxford.

Mackeret, F.J.H., Heron, J. \& Talling, J.F. 1978. Water analysis: some revised methods for limnologists. Cumbria: Freshwater Biological (Association Scientific Publication, 39). Ed. Wilson, Son Ltda, Kendall.

Marker, A.F.H., Nusch, H., Rai, H. \& Riemann, B. 1980. The measurement of photosynthetic pigments in freshwaters and standardization of methods: conclusion and recommendations. Archives Hydrobiology Beih 14: 91-106.

Martins, F.C.O. \& Fernandes, V.O. 2007. Estrutura da comunidade de algas perifíticas em substrato natural da lagoa da Universidade Federal do Espírito Santo, Brasil. Neotropical Biology and Conservation 2: 11-20.

Nakai, S., Inoue, Y. \& Hosomi, M. 1999. Growth inhibition of blue-green algae by allelopathic effects of macrophyte. Water Science and Technology 39: 47-53.
Pellegrini, B.G. \& Ferragut, C. 2012. Variação sazonal e sucessional da comunidade de algas perifíticas em substrato natural em um reservatório mesotrófico tropical. Acta Botanica Brasilica 26: 807-818.

Salomoni, S.E. \& Torgan, L.C. 2008. Epilithic diatoms as organic contamination degree indicators in Guaíba Lake, Southern Brazil. Acta Limnologica Brasiliensia 20: 313-324.

Sand-Jensen, K. \& Borum, J. 1991. Interactions among phytoplankton periphyton and macrophytes in temperate freshwaters and estuaries. Aquatic Botany 41: 137-175.

Santos, T.R., Ferragut, C. \& Bicudo, C.E.M. 2013. Does macrophyte architecture influence periphyton? Relationships among Utricularia foliosa, periphyton assemblage structure and its nutrient $(\mathrm{C}, \mathrm{N}, \mathrm{P})$ status. Hydrobiologia 714: 71-83.

Sartory, D.P. \& Grobbelaar, J.U. 1984. Extraction of chlorophyll a from freshwater phytoplankton for spectrophotometric analysis. Hydrobiologia 114: 177-187.

Stevenson, R.J. 1997. Scale-dependent determinants and consequences of benthic algal heterogeneity. Journal of North American Benthological Society 16: 248-262.

Solorzano, L. 1969. Determination of ammonia in natural waters by the phenolhypochlorite method. Limnology and Oceanography 14: 799-801.

Strickland, J.D.H. \& Parsons, T.R. 1960. A manual of seawater analysis. Bulletin Fisheries Research Board of Canada 125: 1-185.

Thomaz, S.M., Bini, L.M. \& Pagioro, T.A. 2004. Métodos em Limnologia: Macrófitas Aquáticas. In: C.E. Bicudo \& D.C. Bicudo (orgs.). Amostragem em Limnologia, Rima, Editora, São Carlos.

Utermöhl, H. 1958. Zur Vervolkommung der quantitative phytoplankton: metodik. Internationale Vereinigung Theoretische und Angewandte Limonologie 9: 1-38.

Vadeboncoeur, Y.\& Steinman,A.D. 2002. Periphyton Function in Lake Ecosystems. The Scientific World Journal 2: 1-20.

Valderrama, G.C. 1981. The simultaneous analysis of total nitrogen and total phosphorus in natural waters. Marine Chemistry 10: 109-112.

van der Hoek, C., Mann, D.G. \& Jahns, H.M. 1997. Algae: an introduction to phycology. Cambridge University Press, Cambridge.

Vercellino, I.S. \& Bicudo, D.C. 2006. Sucessão da comunidade de algas perifíticas em reservatório oligotrófico tropical (São Paulo, Brasil): Comparação entre período seco e chuvoso. Revista Brasileira de Botânica 29: 363-377.

Vymazal, J.\& Richardson, C.J. 1995. Species composition, biomass, and nutrient content of periphyton in the Florida Everglades. Journal of Phycology 31: 343-354.

Wetzel, R.G. 2001. Limnology: Lake and River Ecosystems. 3 ed. Academic Press, Sam Diego.

Willén, E. 1991. Planktonic diatoms - an ecological review. Algological Studies 62: 69-106.

Whittaker, R. H. \& Feeny, P. P. 1971. Allelochemics: Chemical Interactions between Species. Science 26: 757-770. 